\title{
Doenças neuromusculares raras: um retrato da judicialização no Tribunal Regional Federal da 1⿳亠丷⿵冂丶 Região
}

Rare neuromuscular diseases: a portrait of the judicial decisions in Federal Regional Court of the first region

Enfermedades neuromusculares raras: la judicialización en el Tribunal Regional Federal de la 1aㅡ región

Paulo Henrique Silva Costa ${ }^{1}$

Resumo: Introdução: o estudo aborda a judicialização da saúde promovida pelos portadores de doenças neuromusculares raras em face da Política Nacional de Atenção Integral às Pessoas com Doenças Raras. Metodologia: tratou-se de pesquisa exploratória, descritivo-analítica, quali-quantitativa e de base documental secundária. A coleta de dados foi realizada no sítio eletrônico do Tribunal Regional Federal da 1a região. Resultados: foram selecionados 17 julgados entre os anos de 2007 a 2015. Pacientes com Doença de Pompe, Distrofia Muscular e Esclerose Lateral Amiotrófica compreenderam o universo da amostra. A maioria $(94,11 \%)$, dos dados encontrados, solicitava medicamento não padronizado pelo Sistema Único de Saúde e $76,5 \%$ dos processos judiciais foram julgados procedentes pelo tribunal. Conclusão: concluiu-se que a política específica constituída deve mitigar o número de ações judiciais, porém os resultados demonstraram que o paciente portador de doença neuromuscular rara ainda recorre ao Poder Judiciário para a obtenção de seu tratamento.

Palavras-chave: Decisões Judiciais, Doenças Raras, Doenças Neuromusculares

\begin{abstract}
Introduction: the study analyzes lawsuits about health, promoted by people with rare neuromuscular diseases in the face of the National Assistance Care Policy for People with Rare Diseases. Methods: it was an exploratory, descriptive and analytical, qualitative and quantitative study of secondary database. The research was performed on the Federal Regional Court of the 1st region website. It was selected 17 judicial decisions between 2007 and 2015. Results: the patients with Pompe disease, Muscular Dystrophy and Amiotrophyc Lateral Sclerosis were the sample. The majority $(94.11 \%)$ of the data found, requested medication which is not standardized by the Unified Health System and $76.5 \%$ of claims have been upheld by the court. Conclusion: it was concluded that incorporated specific policy must mitigate the number of lawsuits, but the results showed that the rare neuromuscular disease patient still resorts to the courts to obtain treatment.
\end{abstract}

Keywords: Judicial Decisions, Rare Diseases, Neuromuscular Diseases

Resumen: Introducción: el estudio trató de la judicialización de la salud promovida por las personas con enfermedades neuromusculares raras y su relación con la Política Nacional de Atención Integral a Personas con Enfermedades Raras. Metodología: se trata de estudio exploratorio, descriptivo y analítico, cualitativa y cuantitativa de base

\footnotetext{
${ }^{1}$ Fisioterapia Neurofuncional (Abrafin). Fisioterapeuta do Serviço de Reabilitação do Hospital da Criança de Brasília José Alencar. Brasília - Distrito Federal. Brasil. Email: paulohenrique.fisioterapia@gmail.com
} 
secundaria. La recolección de datos se llevó a cabo en el sitio web del Tribunal Regional Federal de la $1^{\underline{a}}$ región. Resultados: fueron seleccionados 17 decisiones judiciales entre 2007 y 2015. La amuestra comprendió los pacientes con enfermedad de Pompe, Distrofia Muscular y Esclerosis Lateral Amiotrofica. La mayoría (94,11\%), de los datos encontrados, solicitaba medicación que no tenía registro en el Sistema Único de Salud y en $76,5 \%$ de las reclamaciones se han confirmado por el tribunal. Conclusión: se concluyó que la política especial debe para mitigar el número de demandas, pero los resultados mostraron que el paciente con enfermedad neuromuscular rara todavía recurre a los tribunales para obtener su tratamiento.

Palabras-Ilave: Decisiones Judiciales, Enfermedades Raras, Enfermedades Neuromusculares

\section{Introdução}

Para o Ministério da Saúde (MS) uma doença é considerada rara quando afeta até 65 pessoas em cada 100.000 indivíduos, ou seja, 1,3 em cada 2.000. A Organização Mundial da Saúde (OMS) e o Centro Belga de Estudos em Cuidados de Saúde estimam a existência de 5000 a 8000 doenças raras (1) (2) (3).

A Política Nacional de Atenção Integral às Pessoas com Doenças Raras, instituída pela Portaria no 199 de 30 de janeiro de 2014 e alterada pela Portaria no 981 de 21 de maio de 2014, aprova as Diretrizes para Atenção Integral às Pessoas com Doenças Raras no âmbito do Sistema Único de Saúde (SUS) e institui incentivos financeiros de custeio (3) (4).

A Portaria no 199 estabelece dois eixos para a classificação das doenças raras. No primeiro, as doenças de origem genética e no segundo, as não genéticas. As doenças neuromusculares, genericamente denominadas para definir afecções decorrentes de acometimentos primários da unidade motora, fazem parte do universo das enfermidades raras e, portanto, também se apresentam a partir de causa genética ou adquirida (não genética) (4) (5).

A unidade motora é composta pelo motoneurônio medular, raiz nervosa, nervo periférico, junção mioneural e músculo. Disfunções em um ou mais desses elementos podem ocorrer nas seguintes doenças: Atrofia Muscular Espinhal2; Poliomielite; Polineuropatias Hereditárias ou Adquiridas, como Charcot-Marie Tooth ${ }^{3}$ e Guillain Barrè,

\footnotetext{
2 Para maiores informações consulte: http://www.atrofiaespinhal.org/

${ }^{3}$ Para maiores informações consulte: http://abcmt.org.br/
} 
respectivamente; Miastenia Grave; Miopatias, como Distrofia Muscular Congênita e de Duchenne ${ }^{4}$, entre outras (5).

Essas doenças provocam disfunções diversas e comprometem a saúde dos indivíduos portadores (5). Eles podem necessitar de medicamentos, insumos, equipamentos e serviços, que porventura não são oferecidos pelo Estado. No regramento nacional sobre saúde para que uma nova tecnologia seja incluída em lista de dispensação pelo SUS são necessárias a concessão de registro pela Agência Nacional de Vigilância Sanitária (ANVISA) e a análise pela Comissão Nacional de Incorporação de Tecnologias (CONITEC) 5 .

A saúde como direito de todos e dever do Estado, positivada em 1988 no Brasil, abre precedentes para que o cidadão a reclame ao Poder Público (6).

A Política Nacional de Atenção Integral às Pessoas com Doenças Raras suscita essas demandas e ratifica o direito social da população 'esquecida', que encontra na lide uma das formas de garantir assistência integral à saúde (4) (7).

Nesse contexto, Vianna (7) afirma que "o juiz torna-se protagonista direto da questão social". [...] O cidadão recorre a ele utilizando a legislação disponível como alternativa para a garantia de direitos. Ele define esse fenômeno como judicialização da política, que no caso da presente análise compreende o Sistema Único de Saúde do Brasil e suas políticas, programas e estratégias próprios.

Fleury (8) defende que a contradição entre o que está assegurado em leis e a realidade institucional provoca a judicialização. E acrescenta que a não distribuição igualitária dos serviços de saúde, entre os indivíduos no país, fortalece o movimento.

Além disso, observa-se que a tutela judicial pode ser tanto de caráter individual, quanto coletiva. No primeiro o cidadão pode recorrer ao juiz e às Defensorias Públicas e no último, ao Ministério Público (8). A análise deste estudo compreende reclamações

\footnotetext{
${ }^{4}$ Para maiores informações consulte: http://acadim.com.br/

${ }^{5}$ Conforme Decreto № 7.646, de 21 de dezembro de 2011, a incorporação de uma tecnologia no SUS é realiza pelo Ministério da Saúde (MS) através da CONITEC, órgão colegiado desse Ministério que atua por meio da emissão de relatórios de recomendação, nas atribuições relativas à incorporação, exclusão ou alteração pelo SUS de tecnologias em saúde, bem como na constituição ou alteração de protocolos clínicos e diretrizes terapêuticas. O relatório da CONITEC, juntamente com o processo administrativo para incorporação é encaminhado ao Secretário de Ciência, Tecnologia e Insumos Estratégicos do MS que, após sua análise, emite a decisão sobre a incorporação, publicada no Diário Oficial da União.
} 
direcionadas à União, sob a forma de tutela individual, através do Tribunal Regional Federal da $1^{\text {a }}$ região $(\operatorname{TRF} 1)^{6}$.

O TRF 1 compreende 14 unidades da federação e pode representar a situação nacional em ações de saúde demandadas contra o Estado brasileiro nos últimos anos. $A$ sede desse tribunal está localizada na capital do país.

Este trabalho objetiva conhecer a demanda dos portadores de doença neuromuscular rara na abrangência do TRF 1. E ainda correlacioná-la à Política Nacional de Atenção Integral às Pessoas com Doenças Raras.

Não existem estudos que analisam a judicialização, no TRF 1, relacionada aos doentes neuromusculares. Essa assertiva ratifica a necessidade de realizar tal investigação para fins de conhecer a necessidade não atendida dessa minoria.

\section{Metodologia}

Tratou-se de pesquisa exploratória, descritivo-analítica, quali-quantitativa e de base documental secundária.

A coleta de dados foi realizada entre agosto e setembro de 2015 através de pesquisa jurisprudencial, no sítio eletrônico do TRF 1, no seguinte universo: acórdãos, arguições, súmulas, decisões monocráticas, súmulas vinculantes e súmulas e acórdãos do Juizado Especial Federal (JEF). Todos os dados estão abertos ao acesso público e livres de impedimentos para a consulta.

As palavras-chave utilizadas para a busca de processos judiciais compreenderam o nome de enfermidades neuromusculares, de medicamentos, equipamentos e insumos, que são apresentados a seguir: Miopatia; Distrofia Muscular de Duchenne, Becker, Cinturas, Facioescapuloumeral, Côngenita e Miotônica; Atrofia Muscular Espinhal; Esclerose Lateral Amiotrófica; Miastenia; Charcot-Marie Tooth; Guillain Barrè; Pompe; Translarna e Myozyme.

Para a inclusão do julgado na análise de inteiro teor foi considerada a demanda diretamente relacionada à saúde da pessoa com doença neuromuscular rara. Foram selecionados acórdãos, decisões monocráticas e acórdãos do JEF de $2^{\text {a }}$ instância

\footnotetext{
${ }^{6}$ Consultar http:// www.trf1.jus.br/
} 
segundo esse critério. Os dados foram sistematizados em aplicativo Microsoft Excel versão 2010. Julgados com matéria alheia foram excluídos do estudo.

Foi realizada também uma revisão de literatura sobre a judicialização da saúde e doenças neuromusculares raras no banco de dados da Scientific Electronic Library Online (SciELO). Foram buscados periódicos por meio dos descritores: judicialização, judicialização/doenças raras e judicialização/doenças neuromusculares.

Nos julgados envolvendo medicamentos foi analisado se esses possuíam registro na ANVISA e se estavam inseridos na Relação Nacional de Medicamentos Essenciais (RENAME) do SUS.

\section{Resultados}

Foram encontrados, inicialmente, 38 julgados de segunda instância entre os anos de 2003 a 2015. Após conhecimento dos processos apenas 17 compreendiam matéria diretamente relacionada às demandas de saúde de pacientes com doenças neuromusculares raras. Quanto ao posicionamento do tribunal foi considerado o paciente litigante, estando esse na posição de recorrente ou recorrido. Os 17 julgados tiveram suas decisões publicadas entre os anos 2007 a 2015.

O gráfico 1 demonstra a quantidade de processos relacionados a pacientes com doença neuromuscular. As demandas correspondem às doenças de Pompe, Distrofia Muscular e Esclerose Lateral Amiotrófica, respectivamente: 64,7\%, 29,41\% e 5,9\%. 
Gráfico 1 - Distribuição dos julgados, em $2^{\underline{a}}$ instância no TRF 1 , de acordo com as doenças neuromusculares raras, no período de 2007 a 2015.

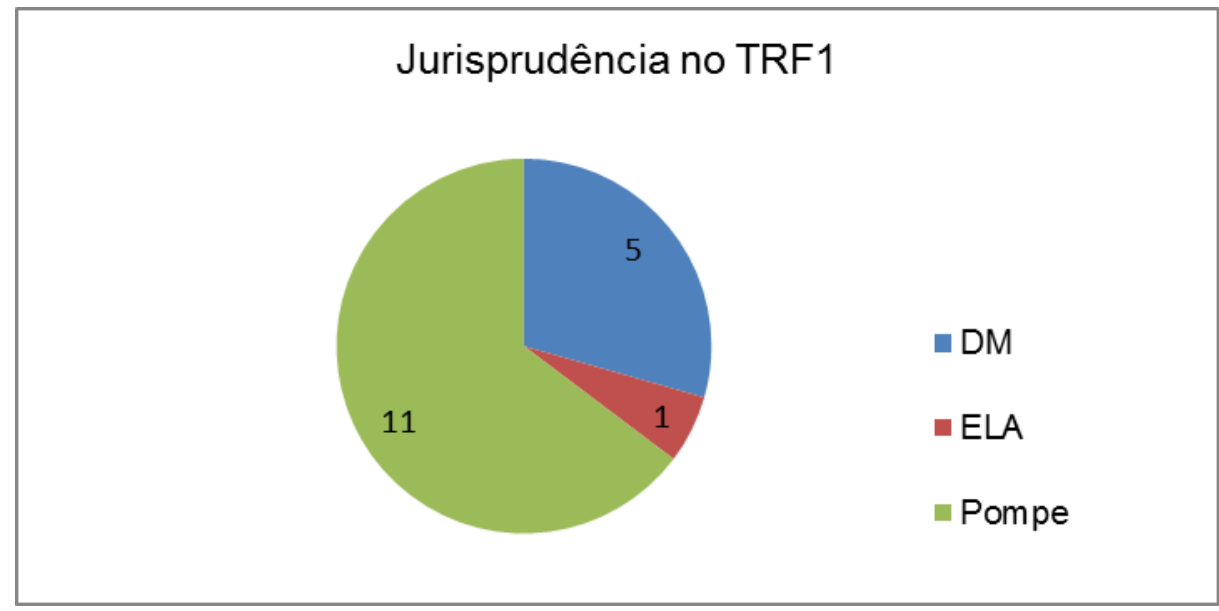

Fonte: Tribunal Regional Federal da 1ㄹ região.

*DM: Distrofia Muscular; ELA: Esclerose Lateral Amiotrófica

O gráfico 2 demonstra o comportamento dos processos judiciais no TRF1 sobre as doenças neuromusculares raras.

Gráfico 2 - Distribuição dos julgados dos processos relacionados a doenças neuromusculares raras, por período de publicação das decisões, entre os anos 2007 e 2015.

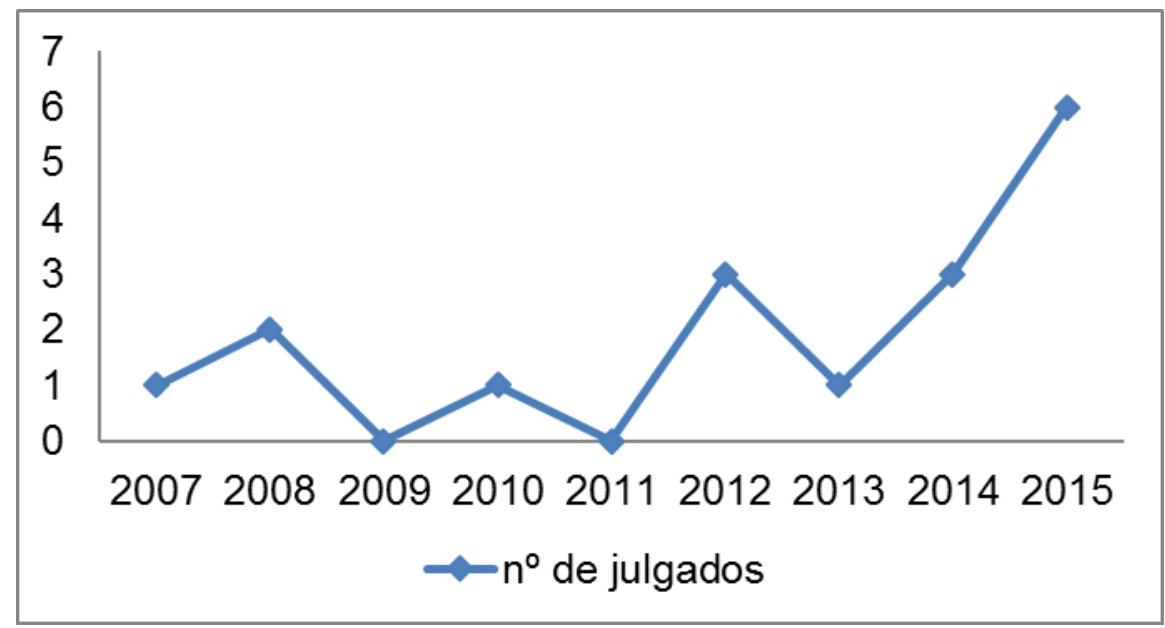

Fonte: Tribunal Regional Federal da $1 \stackrel{a}{ }$ região. 
A soma dos julgados dos anos de 2014 e 2015 corresponde a $58,82 \%$ do total, contrapondo aos demais 7 anos analisados que juntos somam apenas $41,2 \%$.

Em relação ao objeto da lide os medicamentos figuram entre o maior número de demandas. A tabela 1 demonstra a quantidade de medicamentos e procedimentos solicitados e respectiva resposta do Poder Judiciário.

Tabela 1 - Relação do objeto de demanda dos portadores de doenças neuromusculares raras e decisões dos julgados, em 2ª instância no TRF 1, entre os anos de 2007 a 2015.

\begin{tabular}{c|c|c|c|c|c|c}
\hline $\begin{array}{c}\text { Demanda } \\
\text { (Objeto do } \\
\text { litígio) }\end{array}$ & $\begin{array}{c}\text { Demanda } \\
\text { (quantidade) }\end{array}$ & $\%$ & $\begin{array}{c}\text { Decisão } \\
\text { favorável }\end{array}$ & $\%$ & $\begin{array}{c}\text { Decisão } \\
\text { desfavoráve } \\
\text { I }\end{array}$ & $\%$ \\
\hline $\begin{array}{c}\text { Medicamento } \\
\text { TM }^{*}\end{array}$ & 16 & 94,11 & 12 & $75 \%$ & 04 & $25 \%$ \\
& 01 & $\%$ & 01 & $100 \%$ & - & - \\
\hline Total & 17 & $100 \%$ & 13 & $76,5 \%$ & 04 & $24,52 \%$ \\
\hline
\end{tabular}

Fonte: Tribunal Regional Federal da $1^{\text {a }}$ região.

*Transplante de mioblastos ${ }^{7}$

A demanda por medicamento corresponde à maioria do número de julgados, com $94,11 \%$ dos dados analisados. Em um desses era solicitado apenas aumento de dose de medicamento, já concedido pelo TRF 1 em outro pedido.

A tabela 2 demonstra os medicamentos solicitados nos processos judiciais no TRF 1. Foram encontradas demandas relacionadas ao Myozyme, Translarna e Rilutek.

\footnotetext{
${ }^{7}$ Um procedimento que consiste na aplicação, de uma só vez, de 750 injeções de mioblastos (células musculares) saudáveis nos pacientes com Distrofia Muscular de Duchenne. Em 2001 a técnica foi proibida pelo Conselho Federal de Medicina, por não ter resultados comprovados por pesquisa científica. No julgado em análise, que data de 2007, o recorrente (União) teve seu pedido negado e a sentença favorável (dada em 2002) ao paciente foi mantida mediante alegação de o procedimento não causar dano á saúde pública e do fato de o paciente já ter realizado, na ocasião do recurso, a primeira etapa do tratamento.
} 
Tabela 2 - Relação de medicamentos solicitados nos julgados, em $2^{\underline{a}}$ instância no TRF 1 , relacionados a portadores de doenças neuromusculares raras, entre os anos 2007 a 2015. E situação desses fármacos na ANVISA e no RENAME.

\begin{tabular}{cccccc}
\hline DOENÇA & MEDICAMENTO & $\begin{array}{c}\mathrm{N}^{\circ} \\
\text { PEDIDOS }\end{array}$ & $\begin{array}{c}\text { ANO/ } \\
\text { PEDIDO }\end{array}$ & $\begin{array}{c}\text { ANO/ } \\
\text { ANVISA }\end{array}$ & $\begin{array}{c}\text { ANO/ } \\
\text { RENAME }\end{array}$ \\
\hline Pompe & Myozyme & 11 & $2008-15$ & $2007^{8}$ & - \\
DM $^{9}$ & Translarna & 04 & 2015 & - & - \\
ELA $^{*}$ & Rilutek & 01 & 2010 & $2001^{10}$ & $2013^{11}$ \\
\hline
\end{tabular}

Fonte: Tribunal Regional Federal da ${ }^{1}$ a região; sítio eletrônico da ANVISA; Portal da Saúde/Ministério da Saúde.

* Esclerose Lateral Amiotrófica

Entre os 3 fármacos solicitados pelos portadores de DNMs, 2 possuem registro na ANVISA e apenas 1 é padronizado pelo SUS através da RENAME.

O medicamento Myozyme, objeto de 11 julgados entre os anos de 2008 e 2015, possui registro na ANVISA, porém, não faz parte da RENAME. O Translarna, com 4 pedidos em 2015, não possui registro e nem é disponibilizado pela União. Em contrapartida, o Rilutek/Riluzol que foi demandado em 1 julgado, possui registro e já é disponibilizado pelo SUS. Não foram encontrados processos relacionados às demais doenças neuromusculares.

\section{Discussão}

A progressão do número de julgados nos 9 anos analisados evidencia o crescente do fenômeno da judicialização da saúde dos indivíduos com doença neuromuscular no Brasil. O Conselho Nacional de Justiça (CNJ) também observou essa tendência, porém, sob aspecto mais amplo da judicialização da saúde no país através de estudo multicêntrico sobre as relações entre Sociedade, Gestão e Judiciário na efetivação do

\footnotetext{
${ }^{8}$ Registro no 125430020 , obtido em 03/12/2007.

Consultar: http://www7.anvisa.gov.br/datavisa/consulta_produto/rconsulta_produto_detalhe.asp

${ }_{9}$ Nesse caso, deve-se considerar o portador de Distrofia Muscular Duchenne resultante de uma mutação nonsense. É o único indivíduo apto a receber a terapêutica com a medicação, de acordo com o fabricante. Consultar http://www.ema.europa.eu/docs/pt_PT/document_library/EPAR_-

Product_Information/human/002720/WC500171813.pdf

${ }_{10}$ Registro no 113000278 , obtido em 18/07/2001. Nome comercial Rilutek (princípio ativo Riluzol).

Consultar: http://www7.anvisa.gov.br/datavisa/consulta_produto/rconsulta_produto_detalhe.asp

${ }^{11}$ Ano de inclusão do medicamento Riluzol na RENAME.

Consultar http://portalsaude.saude.gov.br/images/pdf/2014/julho/09/livro-rename-2013-atualizado.pdf
} 
direito à saúde. O CNJ realizou levantamento dos processos envolvendo demandas de saúde na esfera pública e da saúde suplementar nos anos 2013 e 2014, em 6 tribunais estaduais do país (9).

Sartori Junior et al (10) também analisaram a judicialização da saúde promovida por pacientes com doença genética rara. Os autores observaram, a partir do banco de dados da Secretaria Estadual de Saúde do Rio Grande do Sul, 13 ações datadas entre 2004 e 2007. Todas estavam relacionadas à terapia de reposição enzimática para a doença de Fabry. A União e o estado foram citados como réu em respectivamente: 1 e 5 processos. E nos outros 7, mais de um ente da federação foi acionado e desses 5 envolveram a União. Todos foram julgados procedentes pelos tribunais.

Em relação ao objeto de litígio 94,11\% correspondem a medicamentos. Nesse universo apenas o Rilutek ou Riluzol estava inserido na RENAME. No entanto, ao comparar o ano em que a decisão do julgado em questão foi publicada e a data de inclusão desse medicamento na RENAME pode-se observar que a padronização só ocorreu após cerca de 3 anos da deliberação favorável ao indivíduo.

A judicialização desses fármacos pode induzir, portanto, a padronização dos mesmos para serem disponibilizados na rede pública de saúde.

Em Minas Gerais, Machado et al (11) também observaram alta prevalência de demandas judiciais por medicamento ao analisar 827 processos entre novembro de 2006 e maio de 2007, na Procuradoria Geral do Estado (PGEMG). Dos 381 medicamentos solicitados $75,5 \%$ não constavam na RENAME, $19,6 \%$ era padronizado pela União e em 4,8\% não havia informação.

Diniz, Machado e Penalva (12), ao analisarem 385 processos da $2^{\underline{a}}$ Vara da Fazenda Pública do Tribunal de Justiça do Distrito Federal e dos Territórios (TJDFT), no período de maio de 2005 a setembro de 2010, observaram que 15\% correspondiam a ações pleiteando medicamentos.

Conti (13) também constata a judicialização de medicamentos não padronizados (REME/DF ${ }^{12}$ e RENAME). A autora verificou que em 2009, 65,9\% dos medicamentos demandados não pertenciam a nenhuma relação de medicamentos oferecidos pelo SUS. Em 2010, esse percentual caiu para 55,2\% e, em 2011 , retomou seu crescimento

12 Relação de Medicamentos Essenciais do Distrito Federal. 
atingindo $65,4 \%$. Ela analisou as ações que citavam a Secretaria de Estado de Saúde do Distrito Federal (SES/DF), através de consulta ao Núcleo de Judicialização da SES/DF e às cópias de processos na Procuradoria Geral do Distrito Federal (PGDF).

Honorato (14) observa um número menor, e tão relevante quanto, ao analisar pedidos judiciais envolvendo medicamentos entre 2010 e 2012. Em um universo de 359 acórdãos do TJDFT relacionados a pedidos de saúde, $37 \%$ compreendiam medicamentos não padronizados.

Informações mais atuais confirmam essa tendência. Magalhães (15) analisou processos julgados (ou em julgamento) entre janeiro e julho de 2014 pelo TJDFT, através dos processos suplementares autuados na PGDF, e observou que $11,54 \%$ das ações pleiteavam medicamentos, e desses, $62,05 \%$ não eram padronizados pelo SUS.

$O$ desequilíbrio entre o surgimento de inovações tecnológicas relacionadas à saúde e os processos de inclusão de novas terapêuticas no rol do SUS pode justificar esse quadro (16). Os medicamentos são inseridos no mercado nacional somente após concessão de registro pela ANVISA. E para seu fornecimento pelo SUS ainda são necessárias avaliação da CONITEC e padronização em listas oficiais.

O tempo para que esses processos ocorram pode interferir na sobrevida de um paciente que tem o medicamento não padronizado (e algumas vezes ainda não registrado pela ANVISA) como a única opção de tratamento. Isso o motiva a recorrer ao Poder Judiciário, amparado pelo 'direito à vida' assegurado pela Constituição Federal de 1988 (6).

Conti (13) resume: "verifica-se estrutura e fluxo insuficientes para promoção do diálogo entre os poderes executivo e judiciário com o objetivo de harmonizar a garantia dos direitos individuais e coletivos à saúde".

Em relação aos medicamentos padronizados e, portanto, fornecidos pelo SUS, foi constatado que após inclusão do fármaco Rilutek/Riluzol na RENAME, em 2013, não foram encontrados processos no TRF 1 que solicitassem tal terapêutica para a ELA. Isso sugere que uma vez aprovado e fornecido pelo Estado, não há judicialização relacionada ao objeto.

Em contrapartida, Delduque e Marques (17) afirmam o contrário ao observar $62,3 \%$ de medicamentos padronizados e apenas $37,7 \%$ não padronizados em uma 
amostra de 87 processos julgados em $1^{\text {a }}$ instância nas Varas de Fazenda Pública do TJDFT, entre os anos de 2005 e 2008. As autoras catalogaram 229 medicamentos nesses processos e todos possuíam registro na ANVISA, o que também difere do estudo no TRF 1.

O Tribunal de Contas do DF (TCDF) afirma, em um relatório de auditoria na assistência farmacêutica na SES/DF em 2011, que "as atividades de programação e de aquisição de medicamentos apresentam falhas graves e não garantem suficientemente o atendimento das necessidades da população" (18). Esse argumento justifica a judicialização mesmo nos casos em que o medicamento já é disponibilizado pelo SUS. Nesses casos já existem vias alternativas ${ }^{13}$ (administrativa e/ou não judicial) para se evitar a lide, porém, seria um tema para outra discussão.

No TRF 1 as decisões favoráveis corresponderam a 76,5\% dos processos analisados, enquanto que em apenas $24,52 \%$ dos julgados o tribunal se posicionou desfavorável ao indivíduo litigante. Conti (13) corrobora com esse achado ao verificar o deferimento da antecipação de tutela em 92,7\% (2009), 90,2\% (2010) e 86,4\% (2011) das ações analisadas. Esse comportamento sugere, em uma primeira análise, que os desembargadores se restringem a garantir o direito à saúde definido na Constituição Federal de 1988 e deixam de analisar aspectos outros como a viabilidade técnica, eficiência e eficácia do produto solicitado.

Em contrapartida, ao comparar o posicionamento do tribunal e o período em que os processos foram julgados foi possível observar nova tendência nas decisões. No TRF 1 entre os anos de 2007 a 2013 as decisões sobre todos os 8 processos foram favoráveis ao indivíduo, enquanto que em 2014 e 2015 dentre os 9 julgados analisados desse período 4 decisões foram desfavoráveis ao doente. Esse posicionamento sugere que o Poder Judiciário vem considerando a existência de diretrizes do SUS antes de determinar o fornecimento da coisa pedida.

É necessário equilibrar o que é determinado na (direitos e garantias para o cidadão) e as diretrizes para o funcionamento do SUS: uma díade de assistência ideal ao indivíduo e o não desajuste nas organizações que prestam a assistência.

\footnotetext{
${ }^{13}$ No DF existe um exemplo: A Câmara Permanente Distrital de Mediação em Saúde, criada pela Portaria Conjunta no 1 de 26 de fevereiro de 2013. Ela integra a SES/DF e a Defensoria Pública do Distrito Federal (DPDF).
} 


\section{Conclusões}

A Política Nacional de Atenção Integral às Pessoas com Doenças Raras foi estabelecida para corrigir lacunas do serviço de saúde prestado a grupos específicos de doentes como os indivíduos com DNM. Para isso suas diretrizes contemplam meios de incentivos e adequações profissionais. Porém, para que haja a implementação efetiva no diagnóstico e terapêutica e transformação do cenário da assistência atual é necessária articulação entre as instâncias administrativas do Estado, e entre profissionais da saúde (3) (4) (19).

A judicialização da saúde promovida pelos portadores de doenças raras influenciou para a criação de uma política específica pelo Poder Executivo. No entanto, ela ainda não abrangeu todos os setores da administração pública e da comunidade para que haja uma assistência integral aos indivíduos. O número de processos que demandam a saúde para essa minoria ratifica tal assertiva.

A constituição da política caracteriza um avanço em busca da integralidade no atendimento de pessoas com doenças raras pelo SUS. Resta ao país, então, o dever de implementá-la e a necessidade de assegurar a assistência compatível com a dignidade da pessoa humana.

Conclui-se, portanto, que apesar de a política específica constituída ter o dever de mitigar o número de ações judiciais, os resultados demonstraram que o paciente portador de doença neuromuscular rara ainda recorre ao Poder Judiciário para a obtenção de seu tratamento. O que evidencia a necessidade de estudos, a médio e longo prazo, que analisem a judicialização da saúde, por esses doentes, em face da Política Nacional de Atenção Integral às Pessoas com Doenças Raras. 


\section{Referências}

1 Vrueh R, Baekelandt ERF, Haan JMH. Rare diseases. In: Priority medicines for Europe and the world "A public health approach to innovation". Brussels: World Health Organization; 2013 [Acesso em 17 set 2015]. Disponível em: http:// http://www.who.int/medicines/areas/priority medicines/en/

2 Denis A, Simoens S, Fostier C, Mergaert L, Cleemput I. Policies for orphan diseases and orphan drugs. Brussels: Belgian Health Care Knowledge Centre; 2009 [Acesso em 17 set 2015]. Disponível em: http://ec.europa.eu/health/ph.../policies orphan en.pdf

3 Brasil. Ministério da Saúde. Portaria n 199, de 30 de janeiro de 2014. Institui a Política Nacional de Atenção Integral às Pessoas com Doenças Raras, aprova as Diretrizes para Atenção Integral às Pessoas com Doenças Raras no âmbito do Sistema Único de Saúde (SUS) e institui incentivos financeiros de custeio. Diário Oficial [da] República Federativa do Brasil. 2014 maio 21; Seção 1. p. 44-52 [Acesso em 13 set. 2015]. Disponível em: http://pesquisa.in.gov.br/imprensa/jsp/visualiza/index.jsp?jornal=1\&pagina $=44 \& d a t a=12 /$ $\underline{02 / 2014}$

4 Brasil. Ministério da Saúde. Portaria no 981, de 21 de maio de 2014. Altera, acresce e revoga dispositivos da Portaria no 199, de 30 de janeiro de 2014. Diário Oficial [da] República Federativa do Brasil. 2014 fev. 21; Seção 1. p. 44-54 [Acesso em 13 set. 2015]. Disponível em:

http://pesquisa.in.gov.br/imprensa/jsp/visualiza/index.jsp?jornal=1\&pagina=44\&data=21/ $\underline{05 / 2014}$

5 Reed UC. Doenças neuromusculares. Jornal de Pediatria 78(supl 1): 90-103,2002 [Acesso em 13 set 2015]. Disponível em:

http://jped.com.br/artigodetalhe.aspx?varArtigo=853.

6 Brasil. Constituição da República Federativa do Brasil, 05 outubro de 1988. Brasília, 5 out 1988. [Acesso em 13 set 2015]. Disponível em:

http://www.planalto.gov.br/ccivil 03/constituicao/ConstituicaoCompilado.htm.

7 Vianna LW, Burgos MB, Salles PM. Dezessete anos de judicialização da política. Tempo Social Revista de Sociologia da USP 19(2): 39-85, 2007 [Acesso em 27 set 2015]. Disponível em: http:// http://www.revistas.usp.br/ts/article/view/12547/14324.

8 Fleury S. Judicialização pode salvar o SUS. Saúde em Debate 36(93): 159-162, 2012 [Acesso em 27 set 2015],. Disponível em

http://peep.ebape.fgv.br/sites/peep.ebape.fgv.br/files/A judicializacao pode salvar o $\mathrm{S}$ US Saude em Debate.pdf

9 Brasil. Conselho Nacional de Justiça. Judicialização da saúde no Brasil: dados e experiência. Coordenadores: Felipe Dutra Asensi e Roseni Pinheiro. Brasília: 2015. [Acesso em 06 nov 2015]. Disponível em 
http://www.cnj.jus.br/files/conteudo/destaques/arquivo/2015/06/6781486daef02bc6ec8c1 e491a565006.pdf

10 Sartori Junior D, Leivas PGC, Souza MV, Krug BC, Balbinotto G, Schwartz IVD. Judicialização do acesso ao tratamento de doenças genéticas raras: a doença de Fabry no Rio Grande do Sul. Ciência \& Saúde Coletiva 17(10):2717-2728, 2012 [Acesso em 06 nov 2015]. Disponível em http://www.scielo.br/pdf/csc/v17n10/20.pdf

11 Machado MAA, Acurcio FA, Brandão CMR, Faleiros DR, Guerra AA, Cherchiglial ML, Andrade EIG. Judicialização do acesso a medicamentos no Estado de Minas Gerais, Brasil. Revista de Saúde Pública 45(3):590-598, 2011 [Acesso em 02 nov 2015]. Disponível em http://www.scielosp.org/pdf/rsp/v45n3/2403.pdf

12 Diniz D, Machado TRC, Penalva J. A judicialização da Saúde no Distrito Federal, Brasil. Revista Ciência \& Saúde Coletiva 19(2): 591-598, 2014 [Acesso em 02 nov 2015],. Disponível em: http://www.scielo.br/pdf/csc/v19n2/1413-8123-csc-19-0200591.pdf

13 Conti, MA. Avaliação das demandas judiciais por acesso a medicamentos no Distrito Federal [Dissertação]. Brasília: Mestrado em Ciências da Saúde, Universidade de Brasília; 2013. [Acesso em 02 nov 2015]. Disponível em:

http://repositorio.unb.br/handle/10482/13532.

14 Honorato S. Judicialização da Política de Assistência Farmacêutica: Discussão sobre as Causas de Pedir no Distrito Federal. Cadernos Ibero-Americanos de Direito Sanitário 4(3): 116-141, 2015 [Acesso em 02 nov 2015]. Disponível em http://www.cadernos.prodisa.fiocruz.br/index.php/cadernos/article/view/208/275

15 Magalhães ASF. Atuação do Poder Judiciário do Distrito Federal no fornecimento de medicamentos não padronizados no SUS. Cadernos Ibero-Americanos de Direito Sanitário. 4(3): 3-24, 2015 [Acesso em 02 nov 2015],. Disponível em http://www.cadernos.prodisa.fiocruz.br/index.php/cadernos/article/view/159/269

16 Pandolfo M, Delduque MC, Amaral RG. Aspectos jurídicos e sanitários condicionantes para o uso da via judicial no acesso aos medicamentos no Brasil. Revista de Salud Pública 14(2): 340-349, 2012 [Acesso em 02 nov 2015]. Disponível em

http://www.scielo.org.co/scielo.php?script=sci arttext\&pid=S012400642012000200014\&l $\underline{\mathrm{ng}=\mathrm{e} \text { \&nrm=iso. }}$.

17 Delduque MC, Marques SB. A Judicialização da política de assistência farmacêutica no Distrito Federal: diálogos entre a política e o direito. Tempus Actas de Saúde Coletiva 5(4): 97-106, 2011 [Acesso em 02 nov 2015],. Disponível em http://www.tempusactas.unb.br/index.php/tempus/article/view/1060.

18 Brasil. Tribunal de Contas do Distrito Federal. Quinta Inspetoria de Controle Externo. Divisão de Auditoria de Programas e de Recursos Externos. Relatório de Auditoria: Assistência Farmacêutica da Secretaria de Estado de Saúde do Distrito Federal. 
Brasília, 2011. [Acesso em 02 nov 2015]. Disponível em:

http://www.tc.df.gov.br/ice5/auditorias/TCDF-AuditoriaAssistenciaFarmaceutica-

RelatorioCompleto.pdf

19 Sousa AM, Sá NM. Análise das características e dos preceitos normativos da Política Nacional de Atenção Integral às Pessoas com Doenças Raras. Cadernos IberoAmericanos de Direito Sanitário. 2015 [Acesso em 02 nov 2015], 4(2): 46-66. Disponível em http://www.cadernos.prodisa.fiocruz.br/index.php/cadernos/article/view/152 